\title{
Indecomposable knots and concordance
}

\author{
BY EVA BAYER-FLUCKIGER* \\ University of Geneva \\ AND NEAL W. STOLTZFUS $\dagger$ \\ Louisiana State University
}

(Received 22 June 1982)

\begin{abstract}
Introduction
R. C. Kirby and W. B. R. Lickorish have proved (cf. (4)) that any classical knot is concordant to an indecomposable knot. In the present note we show that this statement is also true for higher dimensional knots: more precisely, for any higherdimensional knot $K$ there exist infinitely many non-isotopic indecomposable simple knots which are concordant to $K$. This, together with the result of Kirby and Lickorish, gives a complete solution of problem 13 of (1).
\end{abstract}

\section{Simple knots and isometric structures}

Kervaire has proved that the concordance group of even dimensional knots is trivial (cf. (2)), so we only need to consider odd-dimensional knots $K^{2 q-1} \subset S^{2 q+1}$, $q>1$. Such a knot $K^{2 q-1}$ is said to be simple if $\pi_{i}\left(S^{2 q+1} \backslash K^{2 q-1}\right) \cong \pi_{\imath}\left(S^{\prime}\right)$ for $i<q$. Levine has proved that every higher-dimensional $(2 q-1)$-knot is concordant to a simple knot (cf. (5)).

An isometric structure is a triple $(L,\langle\rangle, z$,$) where L$ is a free $\mathbb{Z}$-module of finite rank, $\langle\rangle:, L \times L \rightarrow \mathbb{Z}$ is a $\mathbb{Z}$-bilinear, $\epsilon$-symmetric (where $\epsilon= \pm 1$ ) non-singular form (i.e. the adjoint of $\langle$,$\rangle is an isomorphism), z: L \rightarrow L$ is a $\mathbb{Z}$-linear endomorphism such that $\langle z a, b\rangle=\langle a,(1-z) b\rangle$ for all $a, b \in L$.

Two isometric structures are $i s o m o r p h i c$ if there exists a $\mathbb{Z}$-linear isomorphism which is an isomorphism between the forms and also commutes with the endomorphisms.

An isometric structure $(L,\langle\rangle, z$,$) is metabolic if L$ contains a sub $\mathbb{Z}$-module $M$ which is stable by $z$, such that $\operatorname{rank}(L)=2 \operatorname{rank}(M)$ and that $\langle a, b\rangle=0$ for all $a, b$ in $M$. Two isometric structures $L_{1}$ and $L_{2}$ are Witt-equivalent if there exist metabolic isometric structures $N_{1}, N_{2}$ such that $L_{1} \perp N_{1} \cong L_{2} \perp N_{2}$ (where $\perp$ denotes orthogonal sum).

To any odd-dimensional $\operatorname{knot} K^{2 q-1}$ we can associate an isometric structure with $\epsilon=(-1)^{q}$ (cf. (5) and (3)). Levine has proved that two simple $(2 q-1)$-knots, $q>1$, are concordant if and only if the corresponding isometric structures are Wittequivalent (cf. (5)). The isometric structure of a connected sum is the orthogonal sum of isometric structures.

An isometric structure $(L,\langle\rangle, z$,$) is said to be decomposable if L \otimes \mathbb{Z}[1 / a] \cong$ $\left(L_{1} \otimes \mathbb{Z}[1 / a]\right) \perp\left(L_{2} \otimes \mathbb{Z}[1 / a]\right)$ where $L_{1}, L_{2}$ are non-trivial isometric structures and $a=\operatorname{det}(z)$. Otherwise, we say that $(L,\langle\rangle, z$,$) is indecomposable. This definition is$

* Supported by 'Fonds National Suisse de la Recherche Scientifique'.

† Supported by the National Science Foundation. 
motivated by the fact that if two simple knots are isotopic, then the corresponding isometric structures are $S$-equivalent (cf. (6)) and therefore $\mathbb{Z}[1 / a]$-isomorphic (cf. (9)).

Therefore it suffices to prove the following:

TheOREM. Every isometric structure $(L,\langle\rangle, z$,$) is Witt-equivalent to an indecomposable$ isometric structure.

Addendum. The Witt-equivalence class of $(L,\langle\rangle, z$,$) contains infinitely many in-$ decomposable isometric structures which all have distinct characteristic polynomials.

Indeed, every $(-1)^{q}$-symmetric isometric structure can be realized by a simple $(2 q-1)$-knot (cf. (3)) and the characteristic polynomial of the isometric structure is related to the Alexander polynomial of the knot (cf. (8), p. 14) which is an invariant of the isotopy class of the knot.

\section{Proof of the Theorem}

Let $(L,\langle\rangle, z$,$) be an isometric structure. If f \in \mathbb{Z}[x]$, we shall denote $f^{*}(x)=f(1-x)$. By (8), Proposition $1 \cdot 8$, we may assurne that the minimal polynomial $\phi$ of $z$ can be written $\phi=\phi_{1} \ldots \phi_{n}, \phi_{i}^{*}=\phi_{i}, \phi_{i}$ irreducible and $\phi_{i} \neq \phi_{j}$ if $i \neq j$. Let $p$ be a prime such that $\mathbb{Z}_{(p)}[x] /\left(\phi_{\imath}\right)$ is Dedekind $(i=1, \ldots, n)$ that $p$ does not divide $\phi(0)$ and that $p$ does not divide any of the resultants $\operatorname{Res}\left(\phi_{i}, \phi_{j}\right)$. Let $\psi_{1}, \ldots, \psi_{k}$ be polynomials with integral coefficients such that $\psi_{1}=\phi$, that $\psi_{i+1}$ divides $\psi_{i}$ for all $i=1, \ldots, k-1$ and that the product $\psi_{1} \ldots \psi_{k}$ is the characteristic polynomial of $z$. Let $F_{i} \in \mathbb{Z}[x]$ be monic, irreducible polynomials such that $F_{i} \neq F_{i}^{*}$, that $F_{i} \neq F_{j}$ if $i \neq j$ and $F_{i} \equiv \psi_{i} \bmod p^{2}$ for all $i=1, \ldots, k$ (apply the chinese remainder theorem).

Let $H_{i}=\operatorname{Hyp}\left(\mathbb{Z}[x] /\left(F_{i}\right)\right)$ be the hyperbolic isometric structure associated to the polynomial $F_{i}$, i.e. the isometric structure

$$
\left(\mathbb{Z}^{2 n_{i},}\left(\begin{array}{cc}
0 & I \\
\epsilon I & 0
\end{array}\right),\left(\begin{array}{cc}
M_{i} & 0 \\
0 & I-M_{i}^{t}
\end{array}\right)\right)
$$

where $n_{i}=\operatorname{deg}\left(F_{i}\right), I$ is the identity matrix, $M_{i}$ is the companion matrix to $F_{i}$ and $M_{i}^{t}$ is the transpose of $M_{i}$.

Let $H^{\prime}=H_{1} \perp \ldots \perp H_{k}$.

The first step will be to construct an indecomposable, metabolic isometric structure $M$ such that $H_{(p)}^{\prime}$ is an orthogonal summand of $M_{(p)}$ (notice that if $k=1$, i.e. if the characteristic polynomial of $z$ has no repeated factor, then we can take $M=H^{\prime}$ ).

Let $q=p$ be a prime which does not divide $\phi(0), F_{i}(0), F_{i}^{*}(0)$ for all $i=1, \ldots, k$, and does not divide any of the resultants $\operatorname{Res}\left(F_{i} F_{i}^{*}, F_{j} F_{j}^{*}\right)$, Res $\left(\phi_{i}, F_{j} F_{j}^{*}\right)$. Let $F \in \mathbb{Z}[x]$ be a monic irreducible polynomial such that $F=F^{*}$, that $F(0), F^{*}(0)$ are not divisible by $p$ and that

$$
F \equiv \prod_{i=1}^{k} F_{i} F_{i}^{*} \bmod q^{2}
$$

Let $H^{\prime \prime}=\operatorname{Hyp}(\mathbb{Z}[x] /(F))$ be the hyperbolic isometric structure associated to $F$. Set $H=H^{\prime} \perp H^{\prime \prime}$. Notice that $(q H)^{\#}=(1 / q) H$ (if $K$ is a lattice in $\left(V^{q},[],\right)$, then we denote $K^{\#}=\{x \in V$ such that $\left.[x, K] \subseteq \mathbb{Z}\}\right)$.

Let $T=(1 / q) H / q H$, together with the induced torsion isometric structure (cf. (8), Section 2). 
We have $T=T_{H^{\prime}} \perp T_{H^{\prime \prime}}$, where $T_{H^{\prime}}=(1 / q) H^{\prime} / q H^{\prime}, T_{H^{\prime \prime}}=(1 / q) H^{\prime \prime} / q H^{\prime \prime}$. Let $\pi:(1 / q) H \rightarrow T$ be the projection. Multiplication by $q$ gives the isomorphism:

$$
T_{H^{*}} \cong \frac{1}{i=1, \ldots, k} \operatorname{Hyp}\left(\mathbb{Z} / q^{2}[x] /\left(F_{i}\right)\right)=\frac{1}{i=1, \ldots, k}\left(T_{F_{i} F_{i}^{*}}, S_{i}, z_{i}\right) .
$$

Notice that $T_{F_{i} F_{i}^{*}}$ is a free $\mathbb{Z} / q^{2}$-module.

Similarly,

$$
T_{H^{\prime \prime}} \cong \operatorname{Hyp}\left(\left(\mathbb{Z} / q^{2}\right)[x] /(F)\right) \cong \frac{1}{i=1, \ldots, k} \operatorname{Hyp}\left(\left(\mathbb{Z} / q^{2}\right)[x] /\left(F_{i} F_{i}^{*}\right)\right)
$$

This decomposition follows from an analogue of (8), Theorem 3.2, because $q$ does not divide any of the resultants $\operatorname{Res}\left(F_{i} F_{i}^{*}, F_{j} F_{j}^{*}\right)$.

We shall need the following

Claim 2.1. Let $R$ be a commutative ring and $F=R^{m}$. Let $(F, S, w)$ be an isometric structure. Then

is isomorphic to

$$
\left(F \oplus F,\left(\begin{array}{cc}
S & 0 \\
0 & -S
\end{array}\right),\left(\begin{array}{cc}
w & 0 \\
0 & w
\end{array}\right)\right)
$$

$$
\left(F \oplus F,\left(\begin{array}{cc}
0 & I \\
\epsilon I & 0
\end{array}\right),\left(\begin{array}{cc}
w & 0 \\
0 & 1-w^{t}
\end{array}\right)\right) .
$$

Indeed, an isomorphism is given by

$$
\left(\begin{array}{cc}
I & 0 \\
w S & I
\end{array}\right)\left(\begin{array}{cc}
S^{-1} & 0 \\
0 & I
\end{array}\right)\left(\begin{array}{cc}
I & -I \\
0 & I
\end{array}\right)=\left(\begin{array}{cc}
S^{-1} & S^{-1} \\
w & 1-w
\end{array}\right) .
$$

We shall apply this claim with $R=\mathbb{Z} / q^{2}, F=T_{F_{i} F_{i}^{*}}, S=S_{i}, w=z_{i}$. We obtain:

$$
T_{H^{\prime \prime}} \cong \frac{1}{i=1, \ldots, k}\left\{\left(T_{F_{i} F_{i}^{*}}, S_{i}, z_{i}\right) \perp\left(T_{F_{i} F_{i}^{*}},-S_{i}, z_{i}\right)\right\}
$$

Accordingly, we have a decomposition of $T$

Let

$$
T=\frac{1}{i=1, \ldots, k}\left(T_{i} \perp T_{i} \perp\left(-T_{i}\right)\right) \quad \text { with } \quad T_{i}=\left(T_{F_{i} F_{i}^{*}}, S_{i}, z_{i}\right)
$$

Set

$$
X_{i}=\left\{(x, q y, x) \in T_{i} \perp T_{i} \perp\left(-T_{i}\right)\right\} \quad \text { and } \quad X=\bigoplus_{i=1}^{k} X_{i}
$$

$$
M=\pi^{-1}(X) \subset \frac{1}{q} H
$$

As $X$ is a metabolizer in $T$, we have $M^{\#}=M$. Clearly $M$ is metabolic (cf. (8), proposition 1-3). Moreover, $M_{(q)}$ is indecomposable (where $M_{(q)}=M \otimes \mathbb{Z}_{(q)}$ ). Indeed, assume that $M_{(q)}=M_{1} \perp M_{2}$. Since the decomposition is orthogonal, we may assume that $F F^{*}$ divides the characteristic polynomial of $M_{2}$. Then $M_{1} \subseteq \operatorname{Ann}_{G}\left(M_{(q)}\right)$, where $G=\left(F_{1} \ldots F_{k}\right)\left(F_{1} \ldots F_{k}\right)^{*}$. We have $M_{1} \subset(1 / q) H_{(q)}^{\prime}$, but $X \cap \pi\left((1 / q) H_{(q)}^{\prime}\right)=\{0\}$, therefore $M_{1} \subset q H_{(q)}^{\prime}$. This contradicts the assumption that $M_{1}^{\#}=M_{1}$. Finally, it is clear that $M_{(r)}=H_{(r)}$ if $r \neq q$ because $M \subset(1 / q) H$.

Let $T^{\prime}=(1 / p)\left(L_{ \pm} M\right) / p(L \pm M)=T_{L} \pm T_{M}$, and let $\rho:(1 / p)(L \pm M) \rightarrow T^{\prime}$ be the projection. Let $L_{i}=\operatorname{Ann}_{\phi_{i}}\left(L_{(p)}\right)$. We have

$$
L_{(p)}=\frac{1}{i=1, \ldots, n} L_{i},
$$

because $p$ does not divide $\operatorname{Res}\left(\phi_{i}, \phi_{j}\right)$ if $i \neq j$. Let $\Lambda_{i}=\mathbb{Z}_{(p)}[X] /\left(\phi_{i}\right)$. Then $L_{i}$ is a torsion free $\Lambda_{i}$-module of finite rank. We have assumed that $\Lambda_{i}$ is Dedekind, therefore $L_{i}$ is 
projective. Moreover $\Lambda_{i}$ is semi-local, so $L_{i}$ is a free $\Lambda_{i}$-module of rank, say, $m_{i}$ (cf. (7), p. 24). We have:

$$
T_{L} \cong \frac{1}{i=1, \ldots, n} L_{i} / p^{2} L_{i} \cong \frac{1}{i=1, \ldots, n}\left(T_{\phi_{i}}, S_{i}^{\prime}, z_{i}^{\prime}\right),
$$

where $T_{\phi_{i}}$ is a free $\mathbb{Z} / p^{2}[X] /\left(\phi_{i}\right)$-module of rank $m_{i}$.

On the other hand, we have:

where $T^{\prime \prime}=H^{\prime \prime} / p^{2} H^{\prime \prime}$.

$$
\begin{aligned}
T_{M} & =\frac{1}{p} M_{(p)} / p M_{(p)} \cong\left(\frac{1}{j=1, \ldots, k} H_{j} / p^{2} H_{j}\right) \perp T^{\prime \prime} \\
& \cong \frac{1}{i=1, \ldots, n}\left[\operatorname{Hyp}\left(\mathbb{Z} / p^{2}[X] /\left(\phi_{i}\right)\right)\right]^{m_{i}} \perp T^{\prime \prime} \\
& \cong \frac{1}{i=1, \ldots, n}\left[\operatorname{Hyp}\left(\mathbb{Z} / p^{2}[X] /\left(\phi_{i}\right)\right)^{\left.m_{i}\right] \perp T^{\prime \prime}}\right.
\end{aligned}
$$

Applying Claim 2.1, we have

$$
\operatorname{Hyp}\left(\mathbb{Z} / p^{2}[X] /\left(\phi_{i}\right)\right)^{m_{i}} \cong\left(T_{\phi_{i}}, S_{i}^{\prime}, z_{i}^{\prime}\right) \perp\left(T_{\phi_{i}},-S_{i}^{\prime}, z_{i}^{\prime}\right) .
$$

This induces a splitting of $T^{\prime}$ :

let

$$
T^{\prime} \cong \frac{1}{i=1, \ldots, n}\left(T_{i} \perp T_{i}^{\prime} \perp\left(-T_{i}^{\prime}\right)\right) \perp T^{\prime \prime}
$$

and

Set

$$
Y_{i}=\left\{(x, p y, x) \in T_{i}^{\prime} \perp T_{i}^{\prime} \perp\left(-T_{i}^{\prime}\right)\right\}
$$

$$
Y=\left[\bigoplus_{i=1}^{n} Y_{i}\right] \oplus p T^{\prime \prime}
$$

$$
N=\rho^{-1}(Y) \subset \frac{1}{p}(L \perp M) .
$$

As $Y$ is a metabolizer in $T^{\prime}$, we have $N^{\#}=N$. It is clear that $N$ is Witt-equivalent to $L \perp M$, because they are Witt-equivalent over the rationals (cf. (8), 1.3 and 1.6 ), so $N$ and $L$ are Witt-equivalent.

Let us check that $N$ is indecomposable. Let $a=\operatorname{det}(z) F_{i} F_{i}^{*}(0) \ldots F_{k} F_{k}^{*}(0) F^{*} F^{*}(0)$. Assume that $N \otimes \mathbb{Z}[1 / a]=\left(N^{\prime} \otimes \mathbb{Z}[1 / a]\right) \perp\left(N^{\prime \prime} \otimes \mathbb{Z}[1 / a]\right)$. Suppose that $F$ divides the characteristic polynomial of $N^{\prime \prime}$. Then $F_{1} \ldots F_{k}$ also divides the characteristic polynomial of $N^{n}$. Indeed, if $F_{i}$ divides the characteristic polynomial of $N^{\prime}$, then

$$
K=\operatorname{Ann}_{F_{i} F_{i}^{*}}(N \otimes \mathbb{Z}[1 / a]) \subset N^{\prime} \otimes \mathbb{Z}[1 / a] .
$$

As $q$ is prime to $a$, to $\operatorname{Res}\left(\phi_{j}, F_{i} F_{i}^{*}\right)$ and to $\operatorname{Res}\left(F_{i} F_{i}^{*}, F_{j} F_{j}^{*}\right)$ for all $j, K_{(q)}$ is an orthogonal summand of $N_{(q)}^{\prime}$. But $N_{(q)}=L_{(q)} \perp M_{(q)}$ and $K_{(q)}$ is contained in $M_{(q)}$; therefore this gives a splitting of $M_{(q)}$ which is impossible. So we have

But

$$
N^{\prime} \otimes \mathbb{Z}\left[\frac{1}{a}\right] \subset \operatorname{Aan}_{\phi}\left(N \otimes \mathbb{Z}\left[\frac{1}{a}\right]\right) \subset \frac{1}{p}\left(L \otimes \mathbb{Z}\left[\frac{1}{a}\right]\right) .
$$

therefore

$$
Y \cap \rho\left(\frac{1}{p} L\right)=\{0\}
$$

so we cannot have $\left(N^{\prime}\right)^{\#}=N^{\prime}$.

$$
N^{\prime} \otimes \mathbb{Z}\left[\frac{1}{a}\right] \subset p\left(L \otimes \mathbb{Z}\left[\frac{1}{a}\right]\right),
$$


Proof of the addendum. The characteristic polynomial of the isometric structure $N$ is $\psi_{1} \ldots \psi_{k} F_{1} \ldots F_{k} F_{1}^{*} \ldots F_{k}^{*} F F^{*}$. It is easy to see that we have infinitely many possibilities for, say, $\boldsymbol{F}_{1}$.

\section{Example}

An explicit illustration of the method is given in the following example:

Consider the direct sum of the isometric structures

and

$$
L_{1}=\left(\mathbb{Z} \oplus \mathbb{Z}, \quad\left(\begin{array}{rr}
0 & 1 \\
-1 & 0
\end{array}\right), \quad\left(\begin{array}{rr}
0 & -1 \\
1 & 1
\end{array}\right)\right)
$$

$$
L_{2}=\left(\mathbb{Z} \oplus \mathbb{Z}, \quad\left(\begin{array}{rr}
0 & 1 \\
-1 & 0
\end{array}\right), \quad\left(\begin{array}{rr}
1 & -2 \\
1 & 1
\end{array}\right)\right)
$$

with minimal polynomials $\phi_{1}(X)=X^{2}-X+1$ and $\phi_{2}(X)=X^{2}-X+2$, respectively.

To find an indecomposable isometric structure by our method, we choose a polynomial $F \equiv \phi_{1} \bmod 4$ and also $F \equiv \phi_{2} \bmod 9$. One choice is $F(X)=X^{2}+35 X+29$, and we let $M=\mathbb{Z}[X] /(F(X))$. We construct the desired indecomposable as a lattice inside the rational vector space spanned by

as follows:

$$
L=\operatorname{Hyp}(M) \perp L_{1} \perp L_{2}
$$

The dual lattice to $6 L$ is $\frac{1}{8} L$ with the quotient, $T$, isomorphic to $L / 36 L$ with the isometric structure obtained by reduction modulo 36. Now $T$ splits orthogonally into its $p$-primary components $T_{2}$ and $T_{3}$ inside which we choose metabolic subgroups in the following manner: Since the endomorphisms of $M$ and $L_{1}$ have the same $\bmod 4$ reduction it follows from Claim $2 \cdot 1$ that

$$
T_{2} \cong \operatorname{Hyp}(\bar{M}) \oplus \bar{L}_{1} \oplus \bar{L}_{2} \cong \bar{L}_{1} \oplus-\bar{L}_{1} \oplus \bar{L}_{1} \oplus \bar{L}_{2}
$$

and we may choose the metabolic subgroup

$$
H_{2}=\left\{(2 x, y, y, 2 z) \in \bar{L}_{1} \oplus-\bar{L}_{1} \oplus \bar{L}_{1} \oplus L_{2}\right\}
$$

inside $T_{2}$. Similarly for $\mathrm{H}_{3} \subset T_{3}$.

The desired indecomposable structure is $N=\pi^{-1}\left(H_{2} \oplus H_{3}\right)$ where

is the projection.

$$
\pi: \frac{1}{6} L / 6 L \rightarrow T=T_{2} \oplus T_{3}
$$

First we give the isometric structure for $\hat{L}=\pi^{-1}\left(H_{2} \oplus 3 T_{3}\right)$ which reveals how $L_{1}$ is 'tied' to $\operatorname{Hyp}(M): \hat{L}=\left(\mathbb{Z}^{8}, \hat{S}, \hat{z}\right)$, where

$$
\hat{S}=\left(\begin{array}{rrrrrrrr}
0 & 1 & 0 & 0 & 0 & 0 & 0 & 0 \\
-1 & 0 & 0 & 0 & 0 & 0 & 0 & 0 \\
0 & 0 & 0 & 0 & 0 & 1 & 0 & 0 \\
0 & 0 & 0 & 0 & -1 & 0 & 0 & 0 \\
0 & 0 & 0 & 1 & 0 & 4 & 0 & 0 \\
0 & 0 & -1 & 0 & -4 & 0 & 0 & 0 \\
0 & 0 & 0 & 0 & 0 & 0 & 0 & 1 \\
0 & 0 & 0 & 0 & 0 & 0 & -1 & 0
\end{array}\right)
$$




$$
\widehat{z}=\left(\begin{array}{rrrrrrrr}
-28 & -129 & 128 & 200 & -32 & -50 & 0 & 0 \\
1 & 29 & 0 & -56 & 0 & 14 & 0 & 0 \\
-14 & -50 & 64 & 71 & -16 & -18 & 0 & 0 \\
0 & 32 & 1 & -63 & 0 & 16 & 0 & 0 \\
0 & 0 & 0 & 0 & 0 & -1 & 0 & 0 \\
0 & 0 & 0 & 0 & 1 & 1 & 0 & 0 \\
0 & 0 & 0 & 0 & 0 & 0 & 0 & -2 \\
0 & 0 & 0 & 0 & 0 & 0 & 1 & 1
\end{array}\right) .
$$

Finally the isometric structure for $N=\left(\mathbb{Z}^{8}, S, z\right)$ :

$$
\begin{aligned}
& S=\left(\begin{array}{rrrrrrrr}
0 & 13 & -20 & -12 & 0 & -9 & 0 & 12 \\
-13 & 0 & 0 & 8 & 6 & 6 & 0 & -8 \\
20 & 0 & 0 & -12 & -9 & -9 & 0 & 12 \\
12 & -8 & 12 & 0 & -3 & 0 & 4 & 0 \\
0 & -6 & 9 & 3 & 0 & 4 & 0 & 0 \\
9 & -6 & 9 & 0 & -4 & 0 & 0 & 0 \\
0 & 0 & 0 & -4 & 0 & 0 & 0 & 9 \\
-12 & 8 & -12 & 0 & 0 & 0 & -9 & 0
\end{array}\right) \\
& z=\left(\begin{array}{rrrrrrrr}
-42 & 537 & 592 & -1515 & 432 & 612 & -256 & -676 \\
9 & -135 & -141 & 365 & -96 & -162 & 56 & 164 \\
-14 & 246 & 241 & -633 & 144 & 306 & -84 & -284 \\
0 & 32 & 21 & -62 & 0 & 48 & 0 & -28 \\
0 & 0 & 0 & 0 & 0 & -1 & 0 & 0 \\
0 & 0 & 0 & 0 & 1 & 1 & 0 & 0 \\
0 & 0 & 0 & 0 & 0 & 0 & 0 & -2 \\
0 & 0 & 0 & 0 & 0 & 0 & 1 & 1
\end{array}\right) .
\end{aligned}
$$

Partial credit is awarded to SPEAKeasy for help in the matrix manipulations.

Notice that $\left(\mathbb{Z}^{8}, S, Z\right) \otimes \mathbb{Z}\left[\frac{1}{2}\right]$ is decomposable. As $\operatorname{det}(z)=3770$, this implies that $N=\left(\mathbb{Z}^{8}, S, z\right)$ is decomposable in the sense of our definition of Section 1. However, $N$ is not $S$-equivalent to an orthogonal sum of non-trivial isometric structures (the $S$ equivalence of isometric structures being defined as the $S$-equivalence of the associated Seifert matrices, of. (6), (10)).

Indeed, $\operatorname{det}(Z)=3770$ is square-free. Trotter has proved that two isometric structures with square-free determinant are $S$-equivalent if and only if they are isomorphic over $\mathbb{Z}_{(p)}$ for all primes $p$ (cf. (10), Corollary $4 \cdot 7 a$ ). Now it is easy to check that $N$ does not decompose in the same way over $\mathbb{Z}_{(2)}$ and $\mathbb{Z}_{(3)}$.

Therefore the simple $(4 q+1)$-knot, $q \geqslant 1$, associated to $N$ is indecomposable.

\section{REFERENCES}

(1) Gordon, C. McA. Problems in knot theory. Knot Theory Proceedings (Plans-sur-Bex). Lecture Notes in Mathematics, no. 685 (1978), 309-311.

(2) Kervaire, M. Les noeuds de dimension supérieure. Bull. Soc. Math. France 93 (1965), 225-271.

(3) KervaIre, M. Knot cobordism in codimension two. Manifolds. Lecture Notes in Mathematics, no. 197 (1970), 83-105.

(4) KIRBY, R. C. and Lickorish, W. B. R. Prime knots and concordance. Math. Proc. Cambridge Philos. Soc. 86 (1979), 437-441. 
(5) Levrne, J. P. Knot cobordism groups in codimension two. Comment. Math. Helv. 44 (1969), 229-244.

(6) Levine, J. P. An algebraic classification of some knots of codimension two. Comment. Math. Helv. 45 (1970), 185-198.

(7) SERre, J.-P. Corps locaux (Hermann, 1968).

(8) Stoltzyus, N. W. Unraveling the integral knot concordance group. Mem. Amer. Math. Soc. 192 (1977).

(9) Trotter, H. F. Homology of group systems with applications to knot theory. Ann. Math. 76 (1962), 464-498.

(10) Trotter, H. F. On S-equivalence of Seifert matrices. Invent. Math. 20 (1973), 173-207. 